\title{
Combining an optimisation-based frequency band identification method with historical data for novelty detection under time-varying operating conditions
}

\author{
Stephan Schmidt ${ }^{\mathrm{a}, *}$, Konstantinos C. Gryllias ${ }^{\mathrm{b}, \mathrm{c}}$ \\ ${ }^{a}$ Centre for Asset Integrity Management, Department of Mechanical and Aeronautical Engineering, University of \\ Pretoria, Pretoria, South Africa \\ ${ }^{b}$ Department of Mechanical Engineering, KU Leuven, Celestijnenlaan 300, 3001 Heverlee, Belgium \\ ${ }^{c}$ Dynamics of Mechanical and Mechatronic Systems, Flanders Make, Belgium
}

\begin{abstract}
Frequency band identification methods are used to identify frequency bands in a signal with much damage information. This information can subsequently be used to enhance the weak damage components and facilitate incipient fault detection. In this work, the frequency band identification problem is formulated as an optimisation problem and is solved in two steps. Firstly, a rough solution is obtained by performing a grid-based search, whereafter a local search algorithm is applied to find the optimal solution. The method is specifically developed for rotating machines operating under time-varying conditions. Additionally, this method is combined with historical data from a healthy machine to perform automatic fault detection. The proposed method is investigated and validated on two experimental datasets that were acquired under time-varying operating conditions, with its benefits highlighted.
\end{abstract}

Keywords:

Diagnostics, Frequency Band Identification, Optimisation, Time-varying operating conditions, Historical data

\section{Introduction}

Damaged mechanical components such as bearings and gears could result in unexpected failures of expensive critical assets such as wind turbines $[1,2]$. It is therefore important to use condition monitoring methods to detect and characterise the damage as early as possible. Vibration-based condition monitoring methods are widely used for gearbox condition monitoring, because the measured vibration signals contain much information related to the instantaneous condition of the machine and it has relatively low costs involved with their implementation $[3,4]$. However, it can be difficult to observe the fault signatures associated with the damaged components, because they can be masked by time-varying operating con-

\footnotetext{
${ }^{*}$ Corresponding author.

Email address: stephan.schmidt@up.ac.za (Stephan Schmidt)
} 
ditions [4-6], impulsive noise [7] and dominant deterministic phenomena such as gear mesh interactions $[8]$.

Impulses generated by the damaged components often excite the structural time-invariant resonances, which results in the diagnostic information to manifest in narrow frequency bands $[9,10]$. It is possible to increase the signal-to-noise ratio of the components-of-interest by amplifying these frequency bands. This is one of the motivations behind using the spectral kurtosis and the kurtogram for fault diagnosis applications $[11,12]$. The kurtogram is used to automatically identify the frequency band that maximises the kurtosis, which can subsequently be used to design a bandpass filter to automatically extract this component [13]. Even though the kurtogram has been successful for bearing [13] and gear diagnostics [10], it cannot distinguish between a single transient and repetitive transients. This could lead to the wrong frequency band to be identified [14]. Hence, various other methods such as the protrugram [15], the improved kurtogram [16], the enhanced kurtogram [17], the optimised spectral kurtosis [18], the infogram [14], the envelope-to-harmonic ratio [19], and the autogram [20] have been proposed as alternatives to the original kurtogram.

The aforementioned techniques do not require any prior knowledge about the kinematics of the machine and are therefore referred to as blind methods [21]. It is possible to incorporate prior knowledge about the kinematics of the machine to determine frequency bands that are optimal to identify specific components (e.g. the Ball-Pass Order of the Outer race (BPOO) component of a bearing). These methods are referred to as targeted methods [21]. Smith et al. [21] compared different frequency band identification methods for fault diagnosis and found that targeted methods perform much better than blind methods. Mauricio et al. [22] developed the IESFOgram to automatically determine the optimal frequency band for calculating the Improved Envelope Spectrum (IES), which has been very successful for bearing fault diagnosis. Schmidt et al. [23] proposed the IFBI $_{\alpha}$ gram, which uses a targeted signal-to-noise ratio feature for bearing and gear fault diagnosis. The distcsgram is another targeted method that uses an indicator developed in Ref. [24] for identifying frequency bands that contain much evidence that the bandlimited signal is generalised Gaussian cyclostationary rather than generalised Gaussian stationary [25].

The aforementioned frequency band identification methods are usually estimated with the Short-Time Fourier Transform (STFT) [11, 26] or the Wavelet Packet Transform (WPT) [13] estimators and therefore the resulting feature planes have fixed resolutions. Hence, Zhang and Randall [27] used genetic algorithms to refine the frequency band that was identified with the kurtogram to improve its performance. Wodecki et al. [28] proposed a method to design the impulse response function of a bandpass filter that maximises the kurtosis of the signal with the progressive genetic algorithm. Blind deconvolution methods use an estimated deconvolution filter to extract the impulsive or cyclostationary information from the signals 
$[3,29]$.

However, many rotating machines operate inherently under time-varying operating conditions (e.g. wind turbines) and could adversely influence the application of the conventional frequency band identification methods. In addition, rotating machines operate in healthy conditions for long periods, which makes healthy data easily available when continuous condition monitoring is performed. We therefore propose a simple method to find the optimal informative frequency band under time-varying operating conditions and combine this with historical data from a healthy machine to perform novelty detection. The performance of this method is demonstrated on experimental datasets that were acquired under time-varying operating conditions.

The layout of the paper is as follows: In Section 2, the proposed method is presented, whereafter it is critically applied and validated in Section 3 on two experimental datasets. Existing methods are applied on the experimental data in Section 4 to highlight the performance of the proposed method. Finally, the paper is concluded in Section 5 with recommendations also made for future work.

\section{Proposed method}

We desire to extract a bandlimited signal $\boldsymbol{x}_{\boldsymbol{f}}$ from the measured vibration signal $\boldsymbol{x} \in \mathbb{R}^{N_{x}}$ with a bandpass filter $\boldsymbol{h}_{\boldsymbol{f}}$ that has a passband $\boldsymbol{f}=\left[f_{l}, f_{h}\right]$. The resulting filtered signal

$$
\begin{aligned}
\boldsymbol{x}_{\boldsymbol{f}} & =\boldsymbol{h}_{\boldsymbol{f}} \otimes \boldsymbol{x}, \\
& =\left[x\left[0 ; f_{l}, f_{h}\right], x\left[1 ; f_{l}, f_{h}\right], \ldots, x\left[N_{x}-1 ; f_{l}, f_{h}\right]\right]^{T},
\end{aligned}
$$

can then subsequently be used in combination with signal analysis methods (e.g. synchronous average, squared envelope spectrum, improved envelope spectrum $[9,14,15])$ for fault diagnosis. We can formulate the informative frequency band identification problem as

$$
\begin{aligned}
\underset{\boldsymbol{f}}{\operatorname{maximise}} & \Psi(\boldsymbol{f} ; \boldsymbol{x}) \\
\text { subjected to } & \Delta f_{\text {min }}-f_{l} \leq 0 \\
& f_{h}-\frac{f_{s}}{2}+\Delta f_{\max } \leq 0 \\
& \Delta f_{l h}+f_{l}-f_{h} \leq 0,
\end{aligned}
$$

with the value for $\min \left\{f_{l}\right\}=\Delta f_{\text {min }}$, the value for $\max \left\{f_{h}\right\}=f_{s} / 2-\Delta f_{\text {max }}$, the minimum allowable bandwidth of the bandpass filter denoted by $\Delta f_{l h}$ and the score assigned to the frequency band $f$ for the signal $\boldsymbol{x}$ is denoted $\Psi(\boldsymbol{f} ; \boldsymbol{x}): \mathbb{R}^{2} \rightarrow \mathbb{R}$. This score function, which aims to quantify the amount of 
fault information in the frequency band $\boldsymbol{f}$, can be blind (e.g. kurtosis) or targeted (e.g. an indicator-ofcyclostationarity) and can be calculated from the raw signal; the cyclic spectrum of the signal or from the order-frequency spectral coherence of the signal. Since the bandpass filter has roll-off effects and it is not expected that the informative frequency band will be close to $0 \mathrm{~Hz}$ or close to the Nyquist frequency $f_{s} / 2, \Delta f_{\min }=50 \mathrm{~Hz}$ and $\Delta f_{\max }=50 \mathrm{~Hz}$ in this work. The minimum bandwidth $\Delta f_{l h}$ was set equal to $20 \mathrm{~Hz}$.

The optimisation problem in Equations (3) - (6) can easily be written in an equivalent unconstrained minimisation form

$$
\underset{f}{\operatorname{maximise}} \kappa(\boldsymbol{f})
$$

by using the penalty method [30], where

$\kappa(\boldsymbol{f})=\Psi(\boldsymbol{f} ; \boldsymbol{x})-r \cdot\left(\max \left\{\Delta f_{\min }-f_{l}, 0\right\}^{2}+\max \left\{f_{h}-f_{s} / 2+\Delta f_{\max }, 0\right\}^{2}+\max \left\{\Delta f_{l h}+f_{l}-f_{h}, 0\right\}^{2}\right)$,

is the unconstrained objective function that needs to be maximised. In Equation (8), $r \in \mathbb{R}$ is a penalty parameter and $\max \{a, b\}$ returns the largest number between $a$ and $b$. The negative term of Equation (8) can be minimised with unconstrained minimisation solvers [31,32]. The $r$ in this work is set equal to $10^{9}$.

\subsection{Score function}

It is desired to identify a frequency band that is optimal for fault diagnosis and therefore it is important to utilise a scoring function that quantifies the amount of fault information in a frequency band $\boldsymbol{f}$. The calculation procedure of blind and targeted features is different under time-varying operating conditions. The calculation of the objective function $\Psi(\boldsymbol{f} ; \boldsymbol{x})$ associated with a blind feature is shown in Figure 1 . If

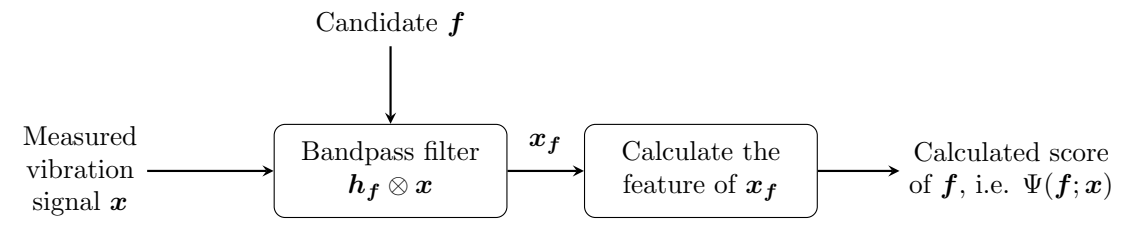

Figure 1: The calculation of the score $\Psi(\boldsymbol{f} ; \boldsymbol{x})$ is shown for the case where the feature is blind, e.g. kurtosis [13] or spectral negentropy [14].

a targeted objective function is used, where the cyclic orders $\boldsymbol{\alpha}$ are targeted, then the objective function $\Psi(\boldsymbol{f} ; \boldsymbol{x}, \boldsymbol{\omega}, \boldsymbol{\alpha})$ needs to be calculated as shown in Figure 2. It is important to perform order tracking to ensure that the features have a consistent representation under time-varying speed conditions and therefore the rotational speed $\boldsymbol{\omega}$ (or instantaneous phase) of the shaft is required as an input. If the speed cannot be measured, it is possible to use tacholess order tracking methods to perform this task [33]. In Figure 2, order tracking is performed after the filtering process to ensure that the angle-time 


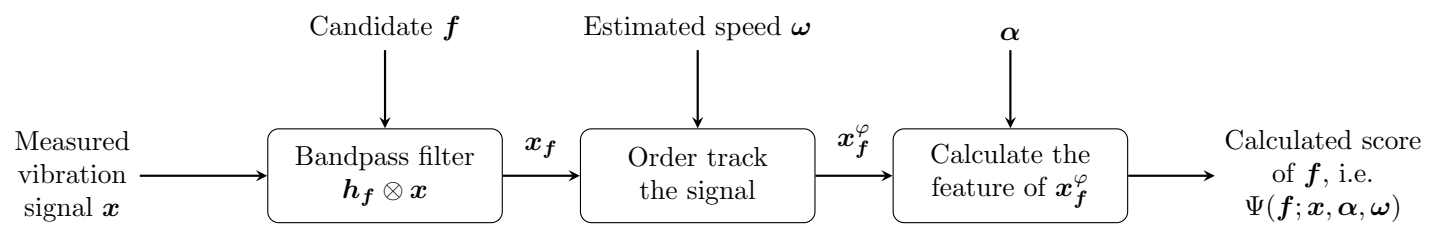

Figure 2: The calculation of the score $\Psi(\boldsymbol{f} ; \boldsymbol{x}, \boldsymbol{\omega}, \boldsymbol{\alpha})$ is shown for the case where the feature (e.g. ICS2 [21]) is targeted on the cyclic orders $\boldsymbol{\alpha}$. The order tracked signal is denoted by the superscript $\varphi$, i.e. $\boldsymbol{x}^{\varphi}$.

cyclostationary characteristics of the impulses are retained [34]. However, performing order tracking in each function evaluation increases the computational cost of the optimisation procedure and therefore if the gearbox is operating under constant or quasi-stationary conditions, the order tracking can be neglected to improve the computational time required for the function evaluations.

The focus of this paper is placed on a signal-to-noise metric that was used in Ref. [9] to estimate the prominence of the cyclic orders $\boldsymbol{\alpha}$ in the SES of a filtered vibration signal. This metric was slightly adapted and used as an integral step in the $\mathrm{IFBI}_{\alpha}$ gram [23], which is a targeted frequency band identification method. The feature

$$
\Psi_{\text {prom }}(\boldsymbol{f} ; \boldsymbol{x}, \boldsymbol{\alpha}, \boldsymbol{\omega})=\sum_{\alpha \in \boldsymbol{\alpha}} \frac{\operatorname{SES}_{\boldsymbol{x}_{f}^{\varphi}}(\alpha)}{\operatorname{noise}\left\{\operatorname{SES}_{\boldsymbol{x}_{f}^{\varphi}}(\alpha)\right\}},
$$

aims to extract the prominence of the cyclic order components $\boldsymbol{\alpha}$ in the signal. The Squared Envelope Spectrum (SES) of the bandlimited signal $\boldsymbol{x}_{\boldsymbol{f}}^{\varphi}$ is denoted by $\operatorname{SES}_{\boldsymbol{x}_{\boldsymbol{f}}^{\varphi}}(\alpha)$ in Equation (9). The SES of the signal $\boldsymbol{x} \in \mathbb{R}^{N_{x}}$ is calculated with

$$
\operatorname{SES}_{\boldsymbol{x}}(\alpha)=\left.\left|\frac{1}{N_{x}} \sum_{n=0}^{N_{x}-1}\right| x[n]\right|^{2} e^{-j 2 \pi n \alpha / f_{s}} \mid
$$

The Squared Envelope Spectrum (SES) highlights the cyclostationary content in the instantaneous power of a signal and is a very popular technique for bearing diagnostics $[8,9]$. This feature calculated with Equation (9) is maximised when the signal components associated with the targeted cyclic orders $\boldsymbol{\alpha}$ are very prominent (i.e. have high signal-to-noise ratios) in the SES. Hence, this feature indicates in which frequency bands it would be easy to identify the cyclic components associated with the damaged component-of-interest.

However, the analytical cyclic orders may differ from the actual cyclic orders of the component-ofinterest due to slip or due to the picket-fence effect and therefore applying Equation (9) directly on the SES of the signal could lead to suboptimal results. Hence, the following feature is calculated instead

$$
\Psi_{\text {prom }}(\boldsymbol{f} ; \boldsymbol{x}, \boldsymbol{\alpha}, \boldsymbol{\omega})=\sum_{\alpha \in \boldsymbol{\alpha}} \frac{\max \left\{\operatorname{SES}_{\boldsymbol{x}_{\boldsymbol{f}}^{\varphi}}\left(\alpha_{e}\right)\right\}_{\alpha \cdot\left(1-c_{t o l} / 2\right) \leq \alpha_{e} \leq \alpha \cdot\left(1+c_{t o l} / 2\right)}}{\operatorname{median}\left\{\operatorname{SES}_{\boldsymbol{x}_{\boldsymbol{f}}^{\varphi}}\left(\alpha_{e}\right)\right\}_{\alpha-1 \leq \alpha_{e} \leq \alpha+1}},
$$


where $\max \left\{\operatorname{SES}_{\boldsymbol{x}_{\boldsymbol{f}}^{\varphi}}\left(\alpha_{e}\right)\right\}_{\alpha \cdot\left(1-c_{t o l} / 2\right) \leq \alpha_{e} \leq \alpha \cdot\left(1+c_{t o l} / 2\right)}$ indicates that the maximum value of the SES in the range $\alpha \cdot\left(1-c_{t o l} / 2\right) \leq \alpha_{e} \leq \alpha \cdot\left(1+c_{t o l} / 2\right)$ is used as an estimate of the amplitude of the componentof-interest. The cyclic order tolerance $c_{t o l}$ is set to $c_{t o l}=0.1$. The denominator of Equation (11) aims to estimate the localised noise level of the SES and uses the median of the SES in the cyclic order band $\alpha-1 \leq \alpha_{e} \leq \alpha+1$. The median is used, because it is more robust to other signal components than the average or the RMS for example [23].

\subsection{Initialisation procedure}

We need to solve the optimisation problem in Equation (7) using numerical optimisation methods. This requires the optimisation solver to be initialised at a specific point in the design space. The objective function is expected to contain more than one local maximum, as the rotating machine may have many natural frequencies in the bandwidth of the measured signal and therefore a random initialisation procedure could result in the optimisation procedure to converge to a local maximum that is not close to the global best.

We therefore propose using a grid-based initialisation procedure to scan the entire frequency range for informative frequency bands. This grid is illustrated in Figure 3. This means that there are several

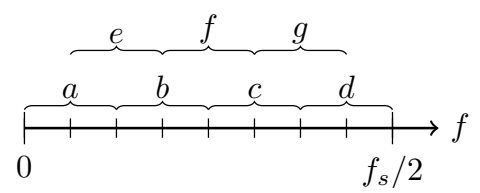

Figure 3: The initialisation procedure is illustrated using seven points evenly spaced between the $0 \mathrm{~Hz}$ and Nyquist frequency $f_{s} / 2 \mathrm{~Hz}$ on the spectral frequency axis $f$. There are seven candidate bandpass filters, denoted $a, b, \ldots, g$ located between the $0 \mathrm{~Hz}$ and $f_{s} / 2 \mathrm{~Hz}$. If $\Delta f=\left(f_{s} / 2\right) / 8$, then the spacing between the centres of the bandpass filters is $\Delta f$ and the bandwidth of the filters is $2 \cdot \Delta f$.

candidate bandpass filters on the grid, with the scoring function (e.g. see Figure 2) being calculated for each candidate bandpass filter. Thereafter, the centre frequency of the bandpass filter with the best score is used to initialise the optimisation algorithm. This initialisation procedure ensures that the region of the optimal informative frequency bands are identified and this ensures the success of the subsequent optimisation procedure. The protrugram used a similar procedure to find the informative frequency bands in the signal [15].

This grid-based initialisation procedure is feasible as the design space is two-dimensional (i.e. $\boldsymbol{f} \in \mathbb{R}^{2}$ ) and the lower and upper bounds are well-defined (e.g. $0 \mathrm{~Hz}$ and $f_{s} / 2$ respectively, where $f_{s}$ is the sampling frequency). We chose the bandwidth of the filter $\Delta f=100 \mathrm{~Hz}$ and the overlap between two consecutive grid points equal to $50 \mathrm{~Hz}$ in the initialisation process. 


\subsection{Optimisation procedure}

Many optimisation methods utilise gradient information to find a local minimum of the cost function. This requires the calculation of the gradient of the derivative of the score function with respect to the design variables, which is not always possible nor feasible [35]. This makes gradient-free search methods such (e.g. Nelder-Mead algorithm [35], particle swarm optimisation [36]) attractive.

Global search methods such as the particle swarm optimisation and the genetic algorithms are generally very computationally expensive to calculate and may not convergence to the global minimum [30, 35]. The Nelder-Mead algorithm is a simple local search method that generally performs well in low-dimensional problems [35, 37]. Since it is assumed that a good initial position is obtained in the previous step and the fact that the optimisation problem is two-dimensional, the Nelder-Mead algorithm is well-suited for this search task.

The Nelder-Mead algorithm is performed by sequentially constructing a sequence of simplexes to improve the cost function under consideration. Four possible operations can be performed on the simplex during the optimisation procedure, namely, reflection, expansion, contraction and shrink [37]. The implementation that is available in the Scientific Python (SciPy) library [32] was used in this work with the standard optimisation parameters being used [37]. The tolerance of the absolute error between iterations was set to $10^{-9}$.

\subsection{Bandpass filtering and signal analysis}

The optimal frequency band $f$ determined in the previous step is used to design a bandpass filter $\boldsymbol{h}_{f}$, whereafter the signal under consideration is filtered with Equation (2) to obtain a vibration signal $\boldsymbol{x}_{\boldsymbol{f}}$ with the fault information enhanced. Subsequently, this filtered signal needs to be further processed to infer the condition of the gearbox. The SES is frequently combined with informative frequency band identification methods (e.g. [15, 21, 23]) for fault detection. The SES of the order tracked filtered signal $[8]$

$$
\operatorname{SES}_{\boldsymbol{x}_{\boldsymbol{f}}}(\alpha)=\mathcal{F}_{\varphi \rightarrow \alpha}\left\{\left|\boldsymbol{x}_{\boldsymbol{f}}^{\varphi}\right|^{2}\right\},
$$

makes it possible to detect periodicities associated with the signal-of-interest in the enhanced vibration signal. The Synchronous Average of the Squared Envelope (SASE) [9, 38, 39]

$$
\operatorname{SASE}(\varphi ; \Phi)=\frac{1}{N_{\Phi}} \sum_{k=0}^{N_{\Phi}-1}\left|x^{\varphi}(\varphi+k \cdot \Phi)\right|^{2},
$$

makes it possible to visualise the modulation that is caused by the damaged component-of-interest with a cyclic period of $\Phi$. The cyclic period $\Phi$ is the reciprocal of the cyclic order of the component-of-interest. 
The SASE of the angular domain signal $x(\varphi)$ is defined over the domain $\varphi \in[0, \Phi)$ and is estimated over $N_{\Phi}$ rotations of the component-of-interest. The SES can be used to identify the cyclic orders of the cyclostationary components, while the SASE can be used to distinguish between localised and distributed gear damage modes.

In the last step of the condition monitoring process, the processed vibration signal is usually converted into a condition indicator, a scalar metric that can be used to detect changes in the condition of the machine. There are many condition indicators available that can be used for gearbox condition monitoring, such as the root-mean-square, kurtosis, indicators-of-cyclostationarity, and methods based on statistical tests $[5,24,40,41]$. In this work, we use the score function of the filtered signal

$$
\mathrm{CI}(\boldsymbol{\alpha})=\sum_{\alpha \in \boldsymbol{\alpha}} \frac{\max \left\{\operatorname{SES}_{\boldsymbol{x}_{f}^{\varphi}}\left(\alpha_{e}\right)\right\}_{\alpha \cdot\left(1-c_{t o l} / 2\right) \leq \alpha_{e} \leq \alpha \cdot\left(1+c_{t o l} / 2\right)}}{\operatorname{median}\left\{\operatorname{SES}_{\boldsymbol{x}_{f}^{\varphi}}\left(\alpha_{e}\right)\right\}_{\alpha-1 \leq \alpha_{e} \leq \alpha+1}}
$$

as a Condition Indicator (CI). This allows us to monitor how the prominence of the component-of-interest changes over time.

\subsection{Novelty detection}

Historical condition monitoring data of a machine operating in a healthy condition is easily acquired when condition monitoring is performed continuously on a machine. This historical data from a healthy machine can therefore be used for automatic fault detection in the form of novelty detection.

We desire to determine a threshold $\tau$ that would allow us to determine whether a processed filtered signal $\mathcal{P}\left\{\boldsymbol{x}_{\boldsymbol{f}}\right\}$ is healthy (i.e., $\mathcal{P}\left\{\boldsymbol{x}_{\boldsymbol{f}}\right\} \leq \tau$ ) or not (i.e., $\mathcal{P}\left\{\boldsymbol{x}_{\boldsymbol{f}}\right\}>\tau$ ), where $\mathcal{P}\{\cdot\}$ denotes a general processing operator (e.g. calculating the SES with Equation (12), the SASE with Equation (13), or the CI in Equation (14)).

In this work, the threshold of the SASE is a scalar variable, i.e. $\tau_{S A S E} \in \mathbb{R}$, while the threshold of the SES is a function of the cyclic order, i.e. $\tau_{S E S}(\alpha) \in \mathbb{R}$. The SASE is compared against a scalar, as the healthy SASE is expected to be relatively flat and aligning the phase between the measurements could be difficult without a keyphasor on the shaft-of-interest. However, irrespective of whether the threshold is a scalar or vector, it is expected to be dependent on the frequency band determined in the previous step, i.e. $\tau(\boldsymbol{f})$. Hence, the following procedure is proposed to determine the threshold for automatic fault detection:

1. Identify a frequency band $f$ that maximises a metric (e.g. Equation (11)) and use this band to design the bandpass filter $\boldsymbol{h}_{\boldsymbol{f}}$. 
2. Use the function $\boldsymbol{h}_{\boldsymbol{f}}$, determined in the previous step, with Equation (2) to filter the vibration signal under consideration and obtain $\boldsymbol{x}_{\boldsymbol{f}}$.

3. For each signal in the healthy historical dataset, use $\boldsymbol{h}_{\boldsymbol{f}}$ determined in the first step, to obtain a filtered healthy signal. Therefore, each healthy signal in the historical dataset will be filtered with the same filter as the signal under consideration.

4. Process the filtered signal with the SASE or calculate a CI based on the data and perform the same processing on the filtered signals from the healthy historical dataset.

5. Use the processed healthy filtered signals to calculate a threshold and compare this threshold to the signal under consideration to perform automatic fault detection.

It is assumed that the healthy machine operated under approximately the same time-varying operating conditions states as the machine under consideration, e.g. that the healthy operating conditions were not all taken during an idling state and the testing signal is from a full load state.

The threshold for the SASE is calculated by firstly calculating the 99th percentile of the SASE of each filtered healthy signal. Thereafter, the maximum value of the 99th percentile of the healthy signals is calculated and used as a threshold. The 99th percentile at each cyclic order value in the SES of the filtered healthy signal is used to set a cyclic order dependent threshold for the SES.

In the next section, the proposed method is investigated on experimental data acquired under timevarying operating conditions.

\section{Experimental investigation}

The gearbox test-rig in Figure 4 is located in the Centre for Asset Integrity Management (C-AIM) laboratory of the University of Pretoria and was used to generate the vibration data considered in this work. The test-rig consists of three helical gearboxes, with the centre gearbox (i.e. Gearbox 2 in Figure

(a) Experimental setup

(b) Back of monitored gearbox
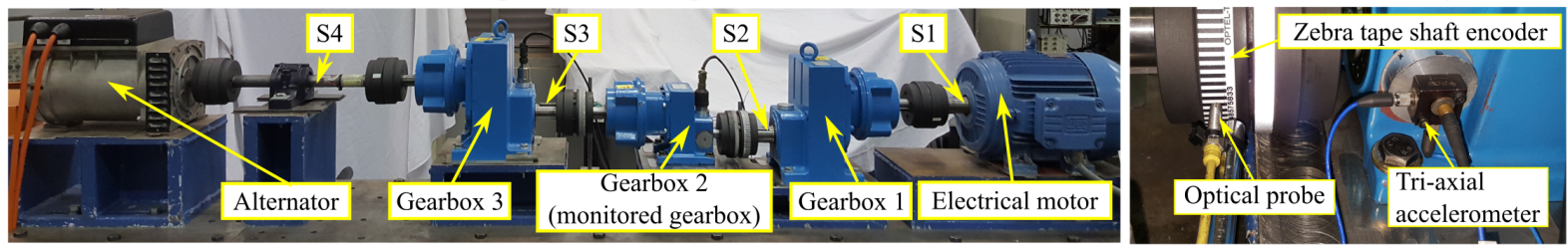

Figure 4: The experimental test-rig that was used to generate the data is presented in (a), with the shafts annotated by $S 1$, $S 2, S 3$ and $S 4$. The tri-axial accelerometer and the optical probe, which are located on the back of the gearbox, are shown in (b).

4(a)) being monitored for damage. The gearbox is instrumented with a tri-axial accelerometer, shown in Figure 4(b), with the axial component of the tri-axial accelerometer being used throughout this paper. The 
vibration signal is sampled at $25.6 \mathrm{kHz}$ and the instantaneous angular speed of the gearbox is estimated with the tachometer signal obtained from the zebra tape shaft encoder and the optical probe highlighted in Figure 4(b). The tachometer signal is sampled at $51.2 \mathrm{kHz}$. All data were acquired with an OROS OR 35 data acquisition system. Since the gear is connected to the shaft with the optical probe, it rotates at 1.0 shaft orders. The modulation from the damaged gear is therefore expected to have a cyclic order of 1.0 shaft orders and therefore the targeted cyclic orders are $\boldsymbol{\alpha}=[1.0,2.0,3.0]$.

The electric motor drives the system and the alternator dissipates the rotational energy, with both being separately controlled with a personal computer. The operating conditions that are investigated in this work are shown in Figure 5.

(a)

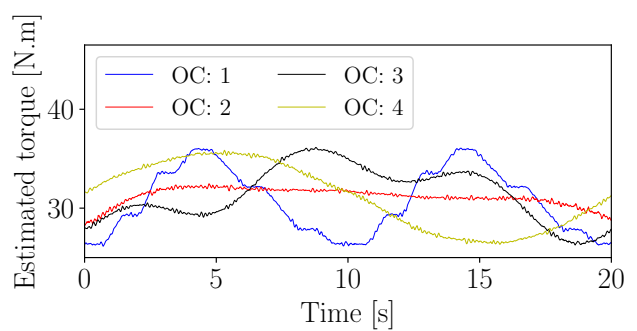

(b)

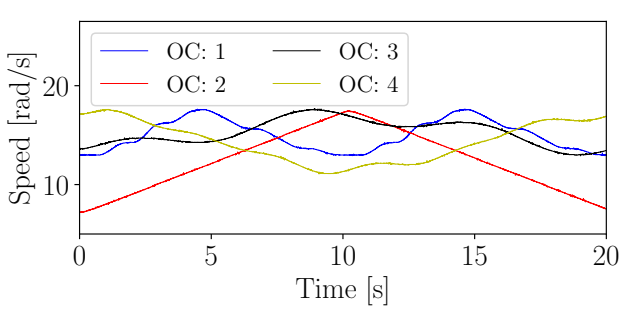

Figure 5: The operating conditions that were estimated at the input shaft of the monitored gearbox. The input shaft of the monitored gearbox is numbered as $\mathrm{S} 2$ in Figure 4.

In this work, the monitored helical gearbox (i.e. gearbox 2 in Figure 4(a)) was damaged with localised and distributed gear damage. Thirty measurements from a healthy gearbox (i.e. with both the pinion and the gear of the monitored gearbox healthy) are used to calculate the thresholds discussed in Section 2.5 and subsequently used for novelty detection.

In the next section, the proposed method is investigated on data that were acquired from the monitored gearbox where the gearbox had localised gear damage.

\subsection{Localised Gear Damage (LGD) investigation}

Localised Gear Damage (LGD) was induced in the gear of the monitored gearbox by seeding a slot in its root, with the seeded slot shown in Figure 6(a). The gearbox was operated for approximately 20 days, with the damaged tooth failing in the final stages of the experiment. In Figure 6(b), the gear is shown after the experiment was completed, with the missing tooth clearly seen. The gearbox was operated under the first operating condition profile in Figure 5 (i.e. OC: 1) during all tests, with 200 measurements acquired between the initial condition (i.e. Figure 6(a)) and the final condition (i.e. Figure 6(b)).

In the first step of the proposed method, the optimisation algorithm needs to be initialised. This is performed by dividing the spectral frequency axis into a grid of points, whereafter the cost function is evaluated at each point on the grid as shown in Figure 3. The result that was obtained from the grid 
(a)

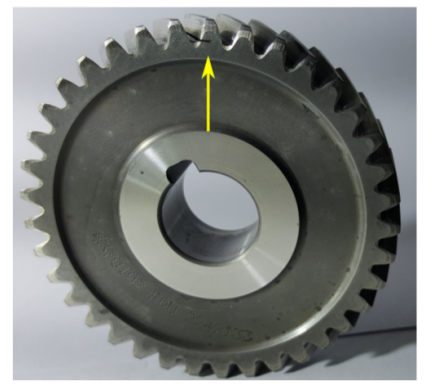

(b)

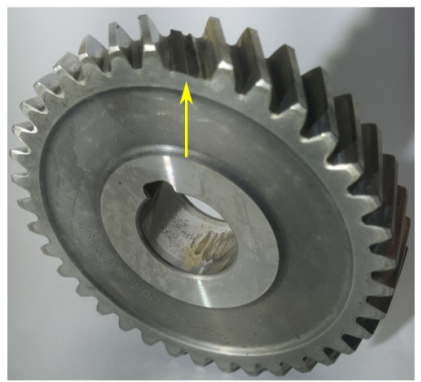

Figure 6: The gear used in the Localised Gear Damage (LGD) investigation is presented. (a) The gear before the experiment started; (b) The gear after the experiment was completed.

(a)

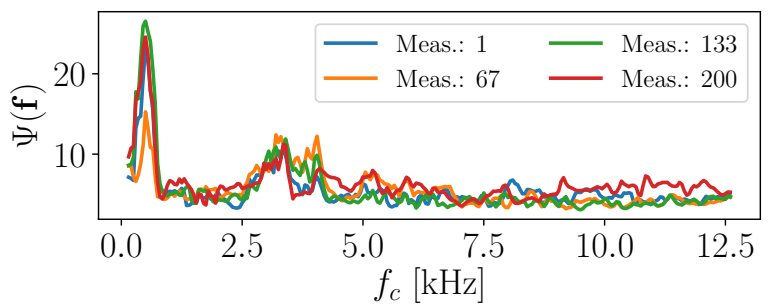

(b)

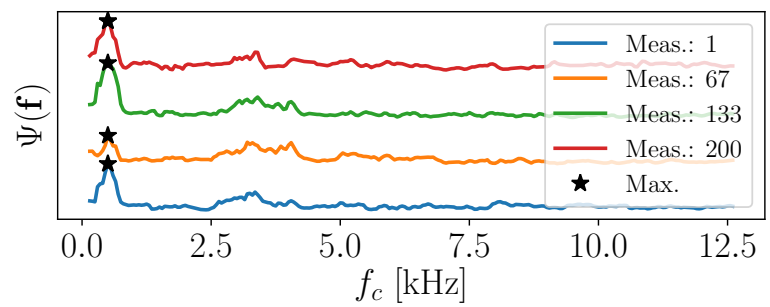

Figure 7: The score function $\Psi(\boldsymbol{f})$, calculated for the frequency band $\boldsymbol{f}=\left[f_{l}, f_{h}\right]$, is presented over the centre frequency $f_{c}=\left(f_{h}+f_{l}\right) / 2$ for four measurements of the localised gear damage experiment dataset. The centre frequency that corresponds to the maximum value of each measurement is used to initialise the optimisation algorithm for that measurement. (a) The score function is presented; (b) The actual score function presented in (a) is given an offset to make the results easier to visualise.

estimation approach is presented in Figure 7 for different measurements in the localised gear damage dataset. The $f_{c}$ that maximises the score is used to centre the initial bandpass filter and the bandwidth was selected to be equal to the bandwidth that was used in the grid-initialisation procedure.

The parameters determined from the initialisation procedure are used to initialise the Nelder-Mead optimisation algorithm for each signal in the dataset, whereafter the Nelder-Mead optimisation algorithm iterated until the change in the design variable $f$ was smaller than the tolerance of $10^{-9}$. The initial value of the frequency band and the final value after completing the optimisation procedure are superimposed on the objective function in Figure 8 for two measurements in the dataset. Even though the initialisation procedure is capable of finding a very good solution, it is not the optimal solution. The optimisation algorithm is capable of finding the optimal frequency band to enhance the signal components associated with the component-of-interest. Since the damage was more developed in Figure 8(b) compared to Figure 8(a), there is a larger region in the design space with fault information. Additionally, the results in Figures 7 and 8 (b) indicate that there can be many local maxima in the design space and therefore it is critical to perform a thorough initialisation process if a local search or optimisation algorithm is utilised.

The SASE and the SES of the raw and filtered signals are compared in Figure 9 for the measurement 
(a)

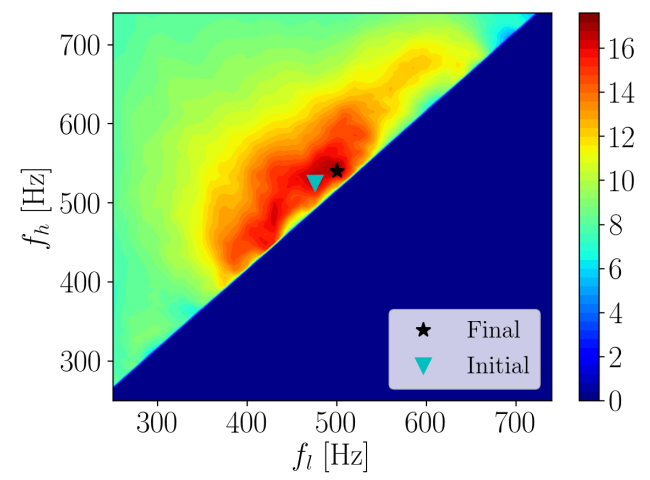

(b)

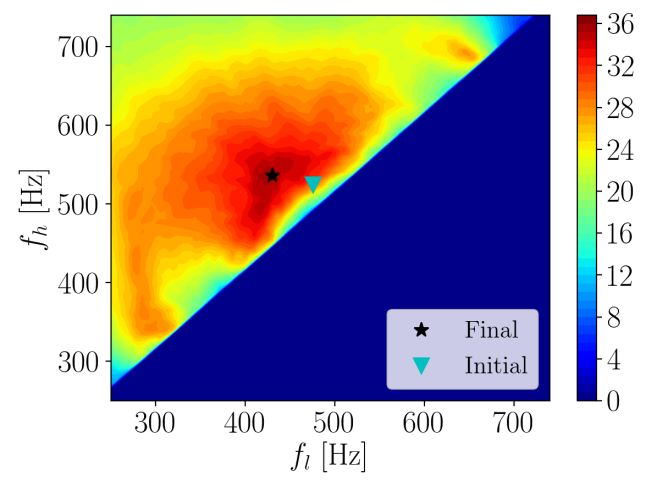

Figure 8: The objective function is shown for two measurements in the localised gear damage experiment, with the initial value and the final value of the optimisation algorithm superimposed on the plot. The initial value is obtained with the initialisation procedure discussed in Section 2.2. In Figures 8(a) and 8(b), the result for the 50th and 75th measurement are respectively shown. The infeasible region was given a value of 0.0 in these plots to make the results easier to visualise. This is possible since the scoring function used in this work is strictly positive.

considered in Figure 8(a). It is not possible to observe the damaged components in the processed raw signals in Figures 9(a) and 9(c). This is attributed to the signal containing dominant impulsive components that are not linked to the condition of the gear, but rather attributed to a floating bearing. The helical gears have larger contact ratios than spur gears, which means that changes in the condition of a single tooth would be more difficult to detect. This makes it difficult to detect the damage in the raw signals.

In contrast, the results in Figures $9(\mathrm{~b})$ and $9(\mathrm{~d})$ indicate that the proposed method is capable of identifying an informative frequency band, which results in the fault information to be enhanced in the vibration signal. The SASE in Figure 9(b) indicates that there is gear damage in the region of 135 degrees, while the SES indicates that the instantaneous power has a strong periodic component with a fundamental order of 1.0 shaft order.

The thresholds, calculated with the procedure discussed in Section 2.5, are superimposed on the SASE and the SES in Figures 9(b) and 9(d) respectively. This makes it possible to automatically determine which signal components are novel and which components are healthy. In Figure 9(b), the SASE exceeds the threshold in the region of 135 degrees, while the cyclic components associated with the gear components (i.e. 1.0 shaft orders and its harmonics) exceeding the threshold in Figure 9(d).

The results of the second measurement considered in Figure 8(b), where the damaged was more developed, is presented in Figure 10. The damage is much more prominent in Figure 10(a) when compared to the previous signal in Figure 9(a). However, the SASE remains adversely affected by the impulsive noise components. In contrast, the SASE of the filtered signal in Figure 10(b) performs much better, with the damaged component very clearly seen in the region of 135 degrees. The threshold makes it possible to automatically identify that this component is damaged and makes it easy to visualise that only a localised 
(a)

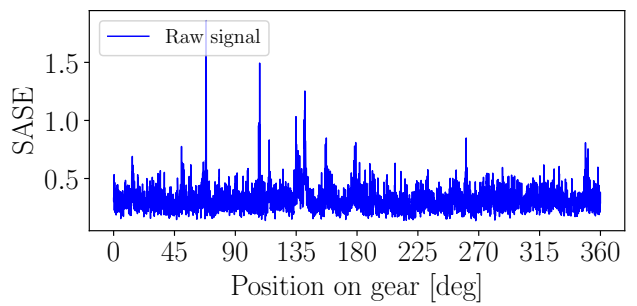

(c)

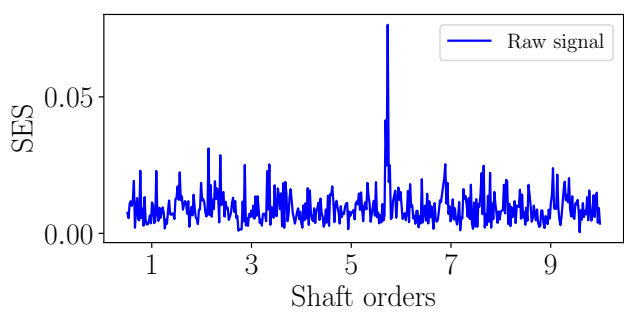

(b)

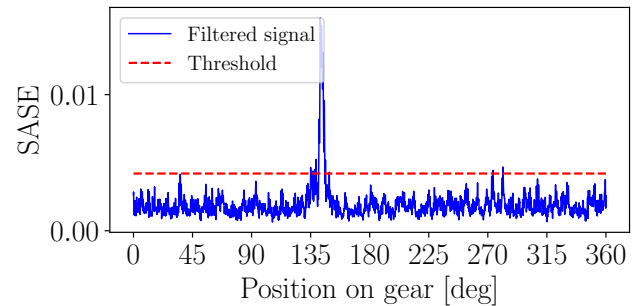

(d)

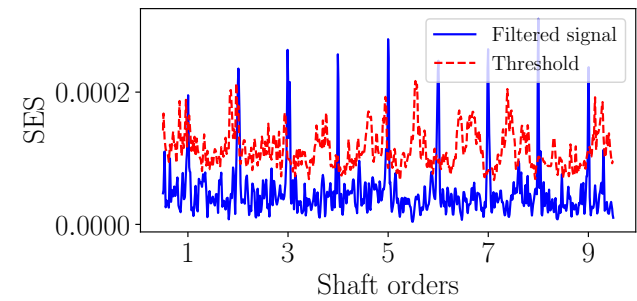

Figure 9: The SASE and SES of the raw and the filtered signals are compared for the 50th measurement of the Localised Gear Damage (LGD) experiment. The threshold was obtained with the procedure discussed in Section 2.5. The objective function of this signal is shown in Figure 8(a).

portion of the gear is damaged. The SES of the raw signal in Figure 10(a) does not contain any evidence that the gear is damaged and is dominated by the strong impulsive component at 5.7 shaft orders. The SES of the filtered signal in Figure 10(d) is very rich with fault information, with the 1.0 shaft order component and its harmonics clearly exceeding the threshold.

The results of ten measurements, evenly spaced over the testing period, are shown in Figures 11 . The SASE and the SES of the raw signals are presented in Figure 11(a) and 11(c). The SES does not contain any indication that the gear is damaged, with only the impulsive noise component at 5.7 shaft orders clearly seen. The SASE in Figure 11(a) is dominated by the noise components, which makes it difficult to detect the gear damage. The SASE and the SES of the filtered signal, shown respectively in Figures 11(b) and 11(d), contain much fault information. The gear damage is clearly seen in the SASE of the filtered signal and it is possible to see changes in the magnitude of the damaged component as the gear degrades. The 1.0 shaft order component and its harmonics are also very prominent in the SES shown in Figure 11(d), with the change in the condition of the gear also clearly seen.

Hence, the filtering process can enhance the damage information and this makes it possible to detect the damaged gear components. In the next section, the performance of the proposed method is investigated on data that were acquired from a gearbox with damage on the surface of the gear. 
(a)

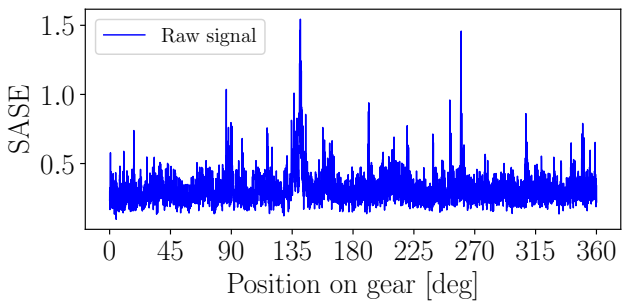

(c)

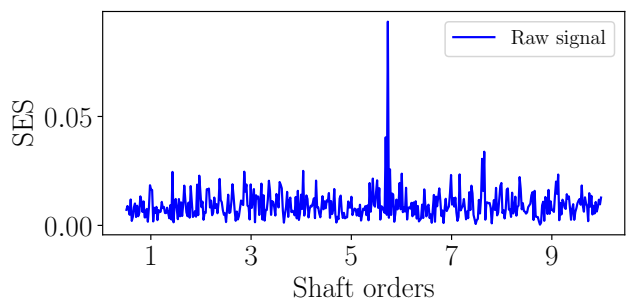

(b)

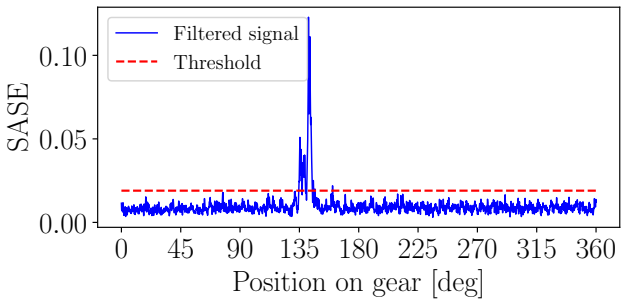

(d)

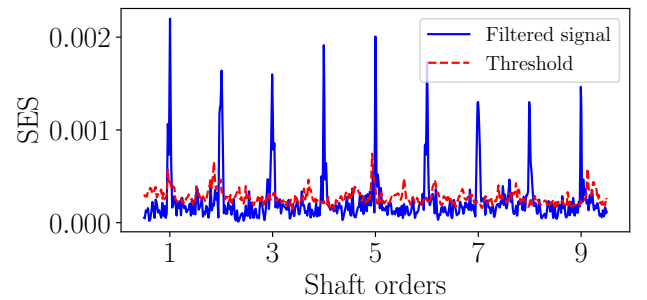

Figure 10: The SES and the SASE are presented for the raw and the filtered signals of the 175th measurement of the Localised Gear Damage (LGD) experiment. The threshold was obtained with the procedure in Section 2.5. The objective function of this signal is shown in Figure 8(b).

\subsection{Distributed Gear Damage (DGD) investigation}

The gear considered in this section is presented in Figure 12. Only half of the gear teeth (i.e. 180 degrees) were damaged, with the damaged region highlighted in Figure 12(a). This makes it possible to determine whether the damaged region can be detected when using a synchronous representation (e.g. SASE). The surface of each gear tooth was damaged with a rotary tool, with the damaged gear teeth shown in Figure 12(b). The gear did not deteriorate significantly during the experiments and therefore the condition of the gear remained approximately constant for the 80 measurements considered in this section. The gears operated periodically under the four operating conditions in Figure 5, i.e. the operating condition sequence was: OC: 1, OC: 2, OC: 3 , OC: 4, OC: 1, OC: 2, etc.

The initialisation procedure was implemented on the dataset to find a good initial position, whereafter the Nelder-Mead algorithm was applied to find the maximum value. The initial value and the final solution, obtained with the optimisation procedure, are superimposed on the objective function in Figure 13 for two measurements in the dataset. Since the damage is relatively small and helical gears are used, the damage components are not very prominent in the signal. This results in a very localised region to contain fault information in the design space shown in Figure 13.

The SASE and the SES results are presented in Figure 14 for one of the measurements of the distributed gear damage experiment. The SASE and the SES of the raw signals do not show convincing evidence that the gear is damaged; the SASE contains much noise over the circumference of the gear in Figure 
(a) Raw

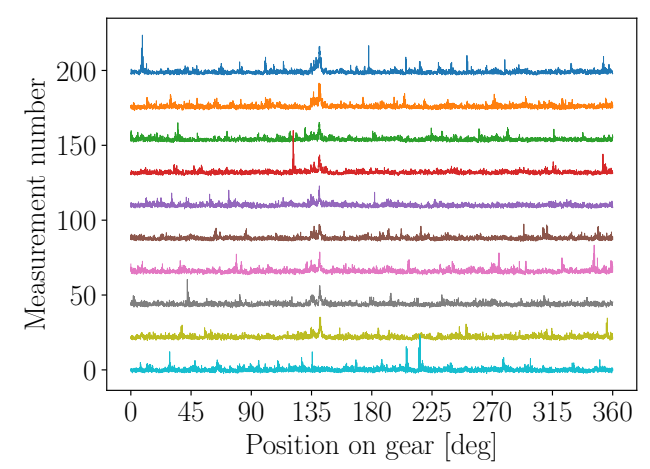

(c) Raw

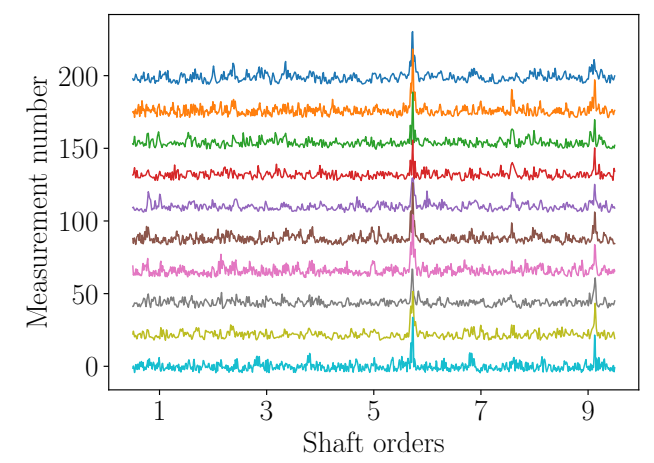

(b) Filtered

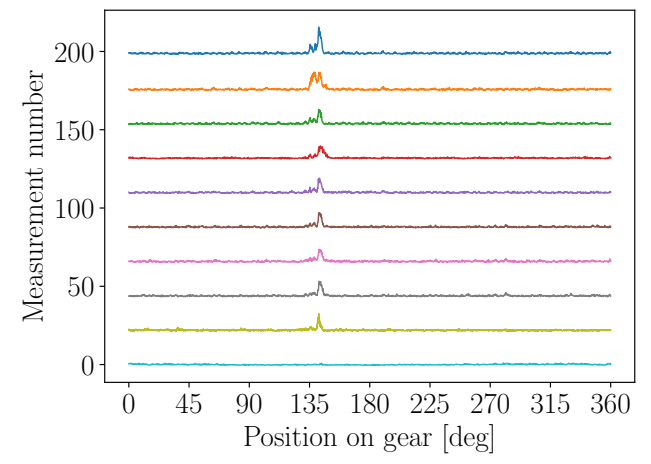

(d) Filtered

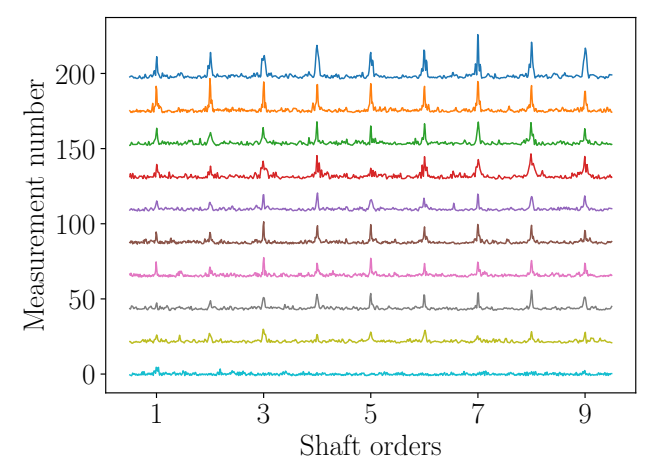

Figure 11: The SASE and the SES are presented over measurement number for the raw and the filtered signals. Since the filtered signals may be associated with different frequency bands and may therefore have different properties (e.g. energy), the SASE and the SES of the filtered signals were normalised with the mean value of the threshold.

14(a) and the SES only contains a feint component at 1.0 shaft order in Figure 14(c). In contrast, the SASE and the SES of the filtered signal display much evidence that the gear is damaged. In Figure 14(b), the SASE exceeds the threshold between 135 and 315 degrees, while the SES in Figure 14(d) shows very dominant components at 1.0 shaft orders and its harmonics. The threshold facilitates damage detection, as it is easier to identify the damaged components when comparing the data to the threshold.

The processed results of ten measurements of the first distributed gear damage experiment are presented in Figure 15 over measurement number. The SASE of the raw vibration signal does not show much evidence that a portion of the gear is damaged. While the SES of the raw signal has a 1.0 shaft order component for some measurements, it does not clearly indicate that it is damaged.

The SASE and the SES of the filtered signal in Figures 15(b) and 15(d) contain much evidence that the gear is damaged; the damaged portion of the gear is observed between 135 and 315 degrees in Figure 15(b) and the 1.0 shaft order component and its harmonics are very prominent in the SES shown in Figure $15(\mathrm{~b})$.

Hence, it is possible to enhance the damaged components in the signal and it is possible to identify the 
(a)

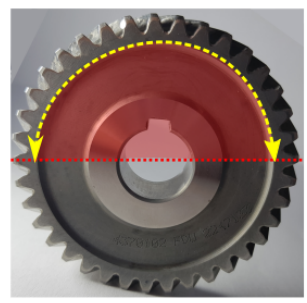

(b)

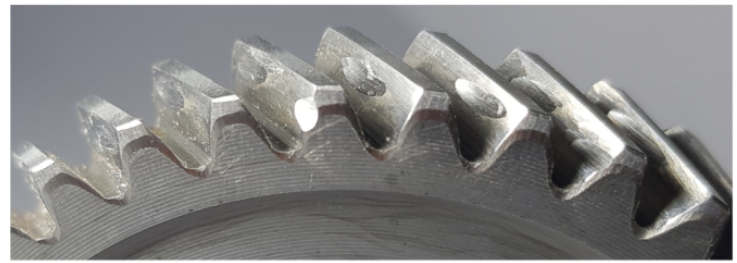

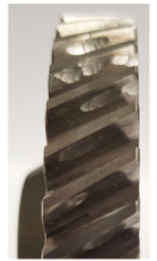

Figure 12: The gear that was tested in the Distributed Gear Damage (DGD) experiment. (a) The portion of the gear that is damaged is highlighted; (b) A zoomed view of the damaged gear teeth is presented.

(a)

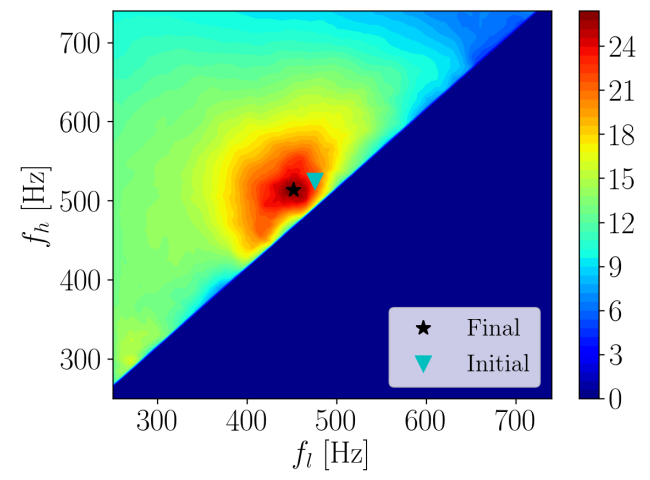

(b)

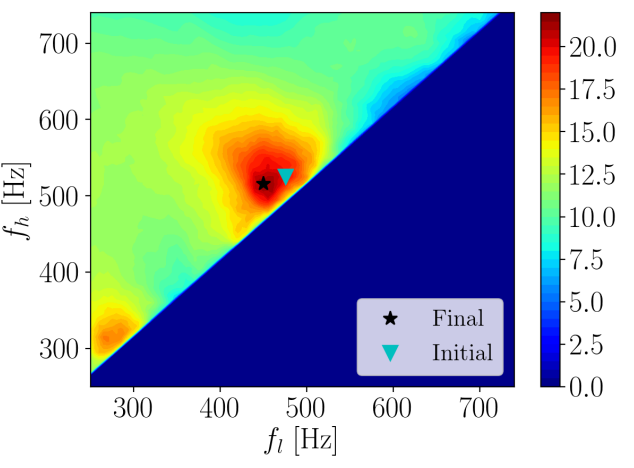

Figure 13: The objective function is shown for two measurements in the distributed gear damage experiment, with the initial value and the final value of the optimisation algorithm superimposed on the plot. In Figures $8(\mathrm{a})$ and 8 (b), the result for the 20 th and 30 th measurement are respectively shown. The infeasible region was given a value of 0.0 in these plots to make the results easier to visualise. This is possible since the scoring function considered in this work is strictly positive.

damaged region of the gear. In the next section, the condition indicator is calculated for the two datasets.

\subsection{Condition Indicator (CI)}

Ultimately, a CI of the processed signal can be used to monitor the condition of the different components of the rotating machine. We calculated the CI in Equation (14) for each measurement in the damaged datasets as well as each measurement in the healthy dataset, with the results presented in Figure 16. At each measurement, the healthy data are filtered with the same filter as the corresponding damaged measurement. This makes the 99th percentile threshold shown in Figure 16 a function of measurement number.

The localised gear damage results in Figure 16(a) indicate that initially the healthy and the damaged condition indicators overlapped. This is attributed to the fact that helical gears were used with a relatively small slot in the teeth. As the gear degrades, the distance between the CI of the damaged measurements and the corresponding CI of the healthy measurements increases, with the damaged measurements exceeding the threshold. 
(a)

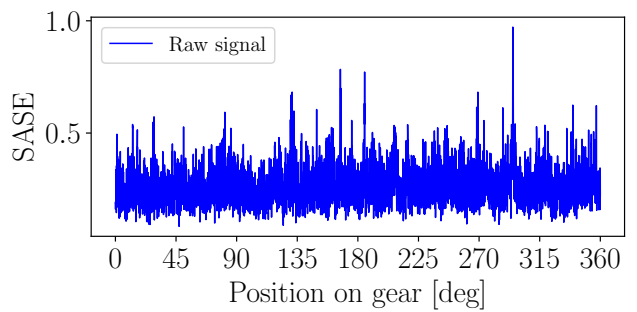

(c)

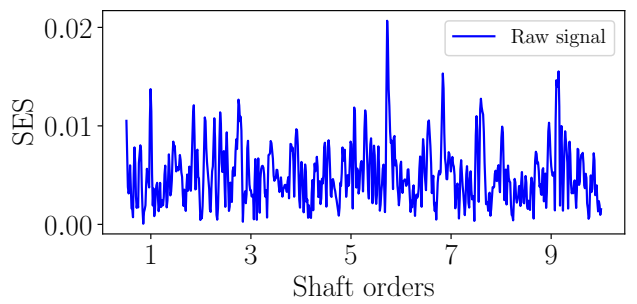

(b)

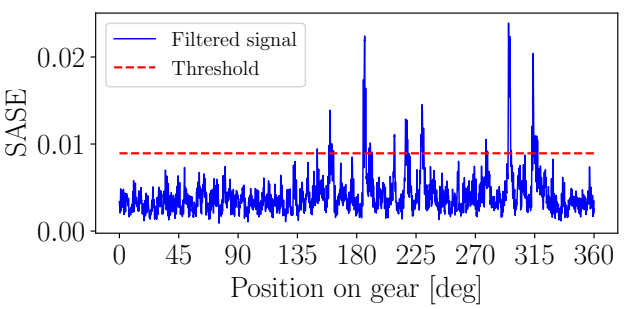

(d)

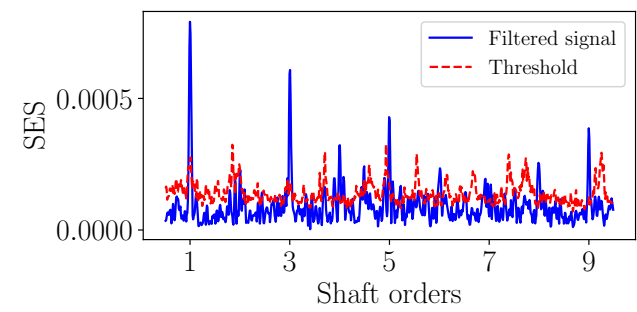

Figure 14: The SES and the SASE are presented for the raw and the filtered signals of the 20th measurement of the Distributed Gear Damage (DGD) experiment. The threshold was obtained with the procedure in Section 2.5. The objective function of this signal is shown in Figure 13(a).

Since the condition of the gearbox remained the same over measurement number for the distributed gear damage case, it would not be possible to see a degrading gear in Figure 16(b). However, the damaged and healthy CI are separated, with the damaged CI exceeding the threshold. Since the bandwidth of the bandpass filter is a function of measurement number (e.g. see Figure 17), the behaviour of the healthy signals and the associated thresholds changes with measurement number.

In the next section, the identified frequency bands, the percentage improvement that is obtained with the optimisation procedure and the computational time are summarised.

\subsection{Summary of results}

The identified frequency bands is shown in Figure 17 as a function of measurement number for the two datasets. Initially, the localised gear damaged dataset was dominated by a frequency band approximately centred at $3.2 \mathrm{kHz}$, whereafter the frequency bands centred approximately at $500 \mathrm{~Hz}$ was identified as the damage progressed. In Figure 17(b), approximately the same frequency band in the region of $500 \mathrm{~Hz}$ was identified for each measurement in the dataset. It is evident from the results in Figure 17 that the informative frequency band can change over time. Since the properties of the signals (e.g. energy) and its condition indicators could be dependent on the frequency band $\boldsymbol{f}$, it makes sense to calculate a new threshold for each signal under consideration with the procedure discussed in Section 2.5.

The percentage improvement

$$
\mathrm{PI}=\left(\kappa\left(\boldsymbol{f}_{f}\right)-\kappa\left(\boldsymbol{f}_{i}\right)\right) / \kappa\left(\boldsymbol{f}_{i}\right),
$$


(a) Raw

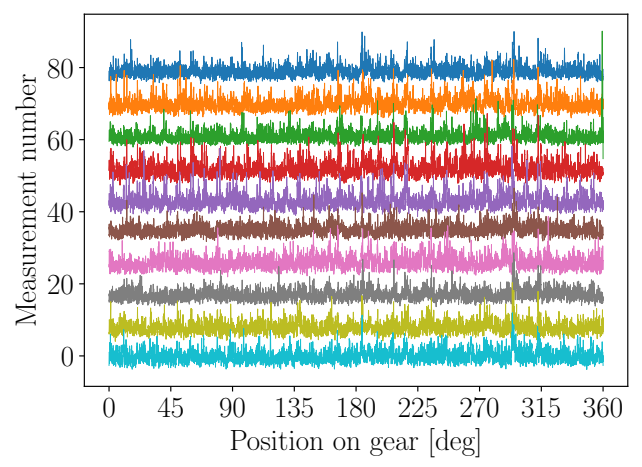

(c) Raw

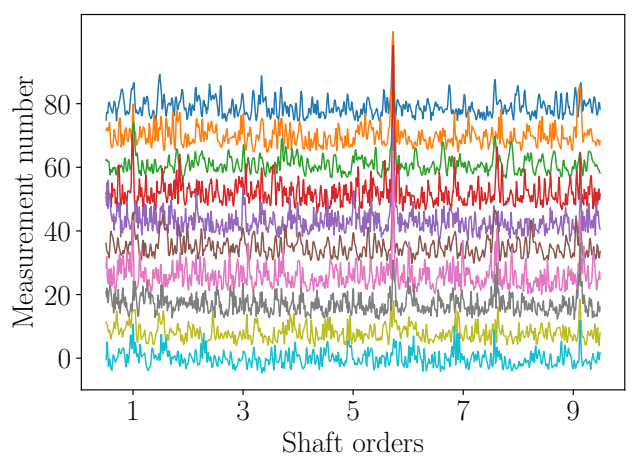

(b) Filtered

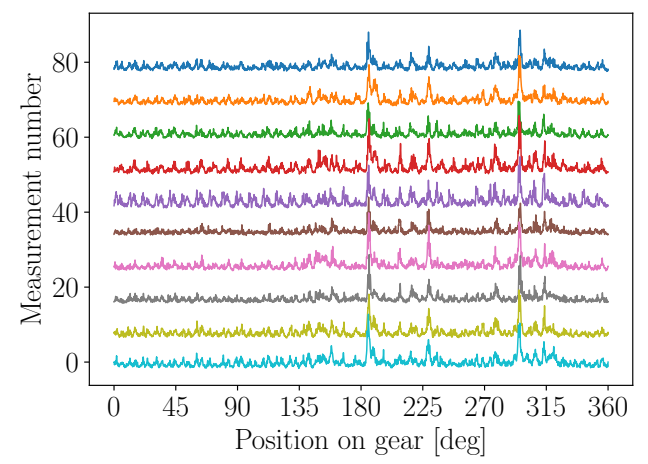

(d) Filtered

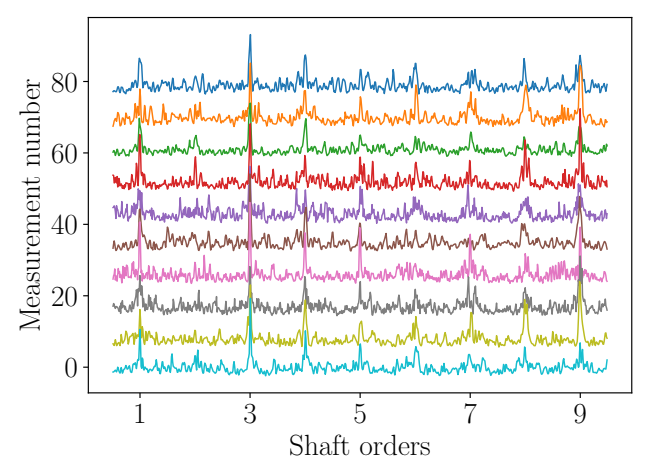

Figure 15: The SASE and the SES are presented over measurement number for the raw and the filtered signals of the distributed gear damage dataset. Since the filtered signals may be associated with different frequency bands and may therefore have different properties (e.g. energy), the SASE and the SES of the filtered signals were normalised with the mean value of the threshold of the signal.

in the objective function when performing the additional optimisation step is presented in Figure 18 for the two datasets. In Equation (15), the unconstrained objective function, defined in Equation (8), is denoted by $\kappa$, the frequency band obtained by the initialisation scheme is denoted by $\boldsymbol{f}_{i}$ and the final frequency band, obtained after the optimisation procedure was completed, is denoted by $\boldsymbol{f}_{f}$. The results in Figure 18 indicates that even though the initialisation scheme can find a good frequency band, the additional optimisation step improved the objective function up to $50 \%$.

The median time of the different steps of the calculation are presented in Table 1. The signal needs to be order tracked in each function evaluation, which makes the function evaluations more expensive to calculate. The initialisation procedure searches the whole frequency range for informative frequency bands, which ensures that a good starting position is used in the optimisation algorithm. However, the computational time is acceptable for condition monitoring applications. 
(a)

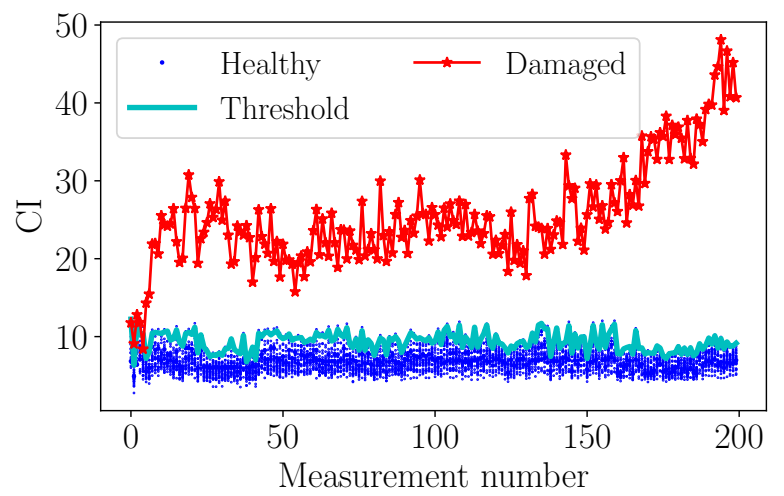

(b)

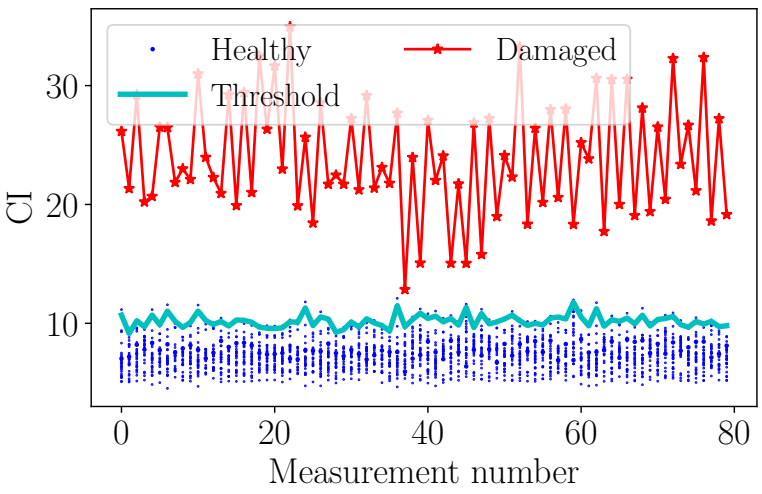

Figure 16: The Condition Indicator (CI), calculated with the filtered vibration signal, is presented for the two damaged gearbox datasets over measurement number. The corresponding CI of the healthy measurements, with its 99th percentile used as the threshold, is presented as well. (a) Localised gear damage experiment; (b) Distributed gear damage experiment.

(a)

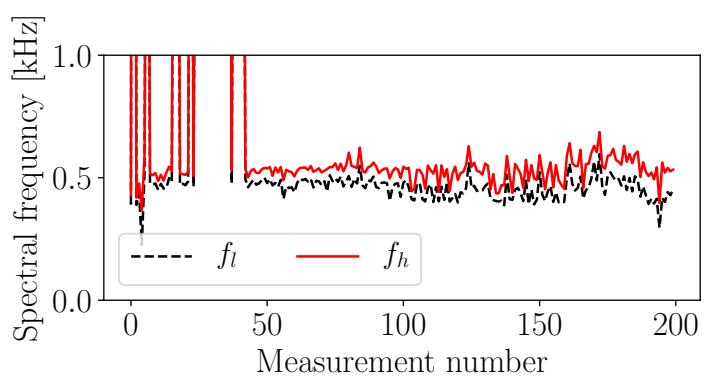

(b)

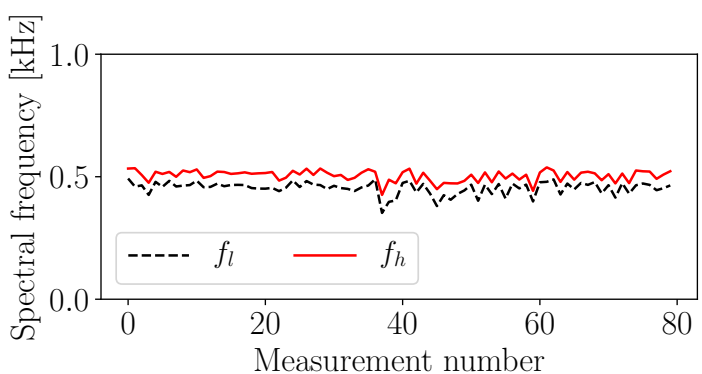

Figure 17: The final frequency band $\boldsymbol{f}=\left[f_{l}, f_{h}\right]$ that was obtained after applying the optimisation algorithm is presented in (a) for the localised gear damage experiment and in (b) for the distributed gear damage experiment.

\section{Comparison with existing methods}

There are many frequency band identification methods available to find informative frequency bands in a vibration signal, e.g. Refs. $[13,14,19,21]$. The kurtogram is a very popular blind frequency band identification method for bearing and gear fault diagnosis $[10,11,13]$ and is therefore considered in this work. The kurtogram is constructed by calculating the kurtosis of different bandlimited signals, with the bandlimited signals usually obtained from the short-time Fourier transform [11] or the wavelet packet transform [13]. The kurtosis is a measure of impulsiveness and can therefore be used to identify frequency bands with much impulsive content. Hence, the frequency band with the most impulsive information would maximise the kurtogram.

The kurtogram, calculated using the short-time Fourier transform-based estimator, is used to identify the frequency band with the most impulsive information for each signal considered in this work. The results of the kurtogram are presented in Figure 19 for the localised and distributed gear damage datasets. Since 
(a)

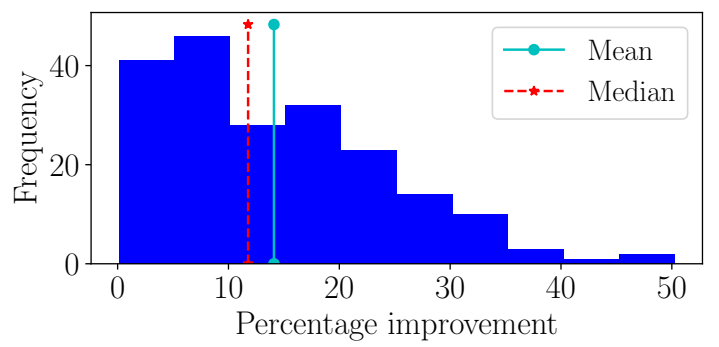

(b)

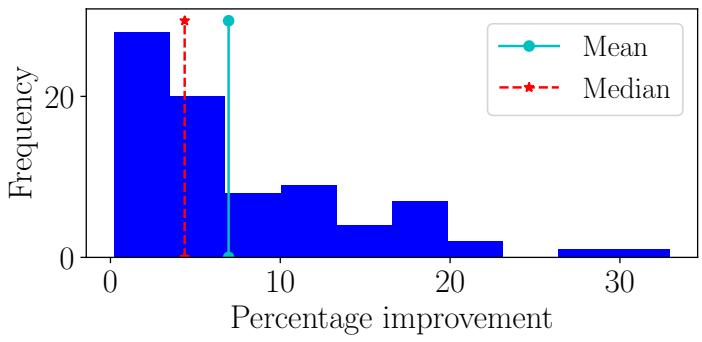

Figure 18: The percentage improvement in the cost function after applying the optimisation algorithm is presented in (a) for the localised gear damage experiment and in (b) for the distributed gear damage experiment. The percentage improvement is calculated with Equation (15).

Table 1: The average time needed to perform the initialisation and optimisation procedures is presented for each measurement in the Localised Gear Damage (LGD) and Distributed Gear Damage (DGD) experiments. The total time is defined as the average duration of both the initialisation and the optimisation parts. The results were calculated in Python 3.7.3 with SciPy 1.3 .0 on a personal computer with $32 \mathrm{~Gb}$ of RAM and an AMD Ryzen 7 2700x eight-core processor.

\begin{tabular}{cccc}
\hline & Initialisation $[\mathrm{s}]$ & Optimisation $[\mathrm{s}]$ & Total $[\mathrm{s}]$ \\
\hline LGD & 45.4809 & 23.8309 & 69.3118 \\
DGD & 62.0048 & 27.7201 & 89.7249 \\
\hline
\end{tabular}

the vibration signals contain much impulsive information not related to the condition of the gears, the kurtogram is maximised by the wrong frequency band. The resulting bandlimited signals do not contain any fault information signals and therefore the damage cannot be detected using the SASE and the SES in Figure 19. The kurtogram is rather maximised by the impulsive noise component with a cyclic order of 5.7, which is very prominent in the SES in Figures 19(b) and 19(d). Since this impulsive component is non-synchronous with the rotation of the gear, it is randomly scattered over the circumference of the gear in Figures 19(a) and 19(c).

However, the kurtogram cannot distinguish between periodic impulses and random impulses and therefore the ICS2gram is investigated. The ICS2gram is a targeted frequency band identification method that uses an indicator-of-cyclostationarity to identify which frequency bands contain cyclostationary information due to damaged machine components [21]. The indicator-of-cyclostationary is calculated for the gear component, i.e. $\boldsymbol{\alpha}=[1.0,2.0,3.0]$, in the different frequency bands of the order tracked vibration signal and is maximised to find the optimal frequency band for fault diagnosis. The vibration signal is order tracked to reduce the smearing of the cyclic components due to changes in the speed of the machine. The SASE and the SES are presented in Figure 20 for the bandlimited signals obtained by the ICS2gram. The results in Figures 20(a) and 20(b) indicate that it is possible to detect the gear damage for only one of the ten measurements, while the distributed gear damage cannot be detected in Figures 20(c) and 20(d).

The condition indicator, defined in Equation (14), is calculated by using the bandlimited signals obtained with the kurtogram and the ICS2gram, with the results presented in Figures 21(a) and 21(b) 
(a) SASE: LGD

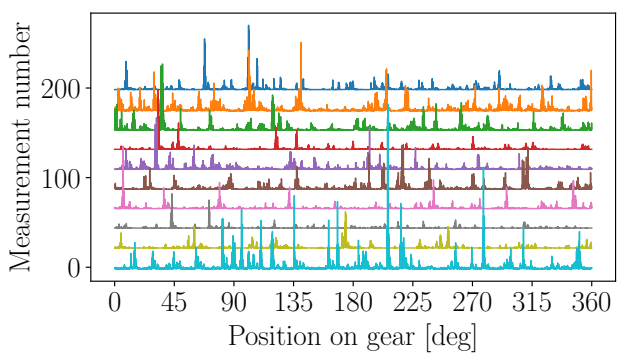

(c) SASE: DGD

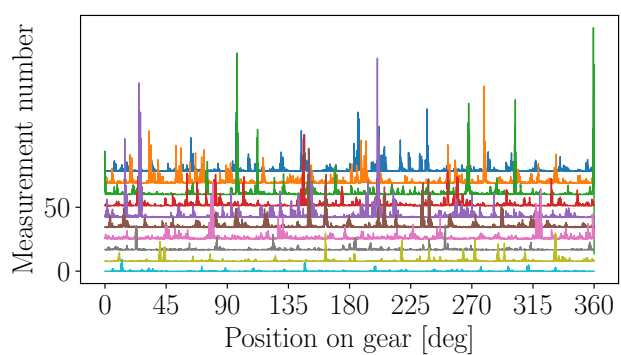

(b) SES: LGD

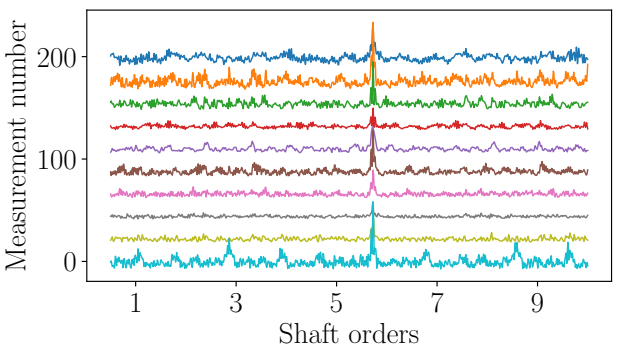

(d) SES: DGD

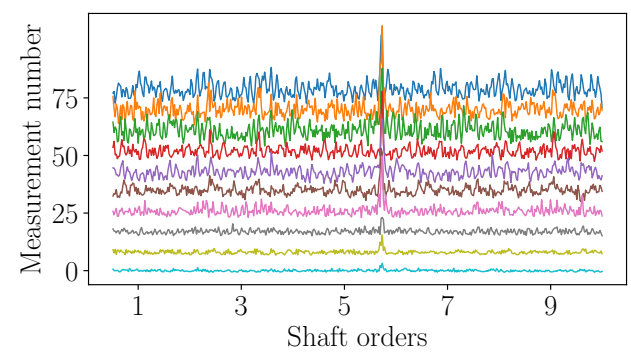

Figure 19: The Synchronous Average of the Squared Envelope (SASE) and the Squared Envelope Spectrum (SES), obtained by using the kurtogram to design a bandpass filter, are presented for the Localised Gear Damage (LGD) and Distributed Gear Damage (DGD) investigations.

respectively for the localised gear damage experiment. The deterioration of the gear is not identified in Figure 21(a) as the kurtogram did not select the appropriate frequency bands for fault diagnosis. Even though the results in Figure 20 indicate that the ICS2gram performed poorly to extract the fault information in the signal, two events are seen in the condition indicator in Figure 21(b). The initial deterioration of the gear tooth is seen in the region of the 25 th measurement and the imminent failure of the gear tooth is seen in the region of the 160th measurement. Even though these two events are clearly seen, the condition indicator fluctuates significantly at these events (i.e. it is not very robust) and the deterioration of the gear tooth is not seen. Hence, the conventional methods fail to detect the gear damage investigated in this work. This is attributed to the dominant impulsive components in the signal and to the use if helical gears, which have larger contact ratios than spur gears, and mask the damaged components.

The computational time of the two methods is summarised in Table 2. Even though the proposed

Table 2: The average time needed to perform find the frequency band with the kurtogram and the ICS2gram for each measurement in the Localised Gear Damage (LGD) and Distributed Gear Damage (DGD) experiments. The results were calculated in Python 3.7.3 with SciPy 1.3.0 on a personal computer with $32 \mathrm{~Gb}$ of RAM and an AMD Ryzen 7 2700x eight-core processor.

\begin{tabular}{ccc}
\hline & Kurtogram & ICS2gram \\
\hline LGD & 12.036 & 13.020 \\
DGD & 11.974 & 13.304 \\
\hline
\end{tabular}


(a) SASE: LGD

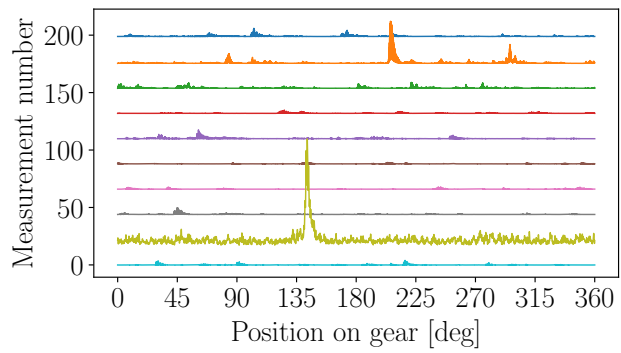

(c) SASE: DGD

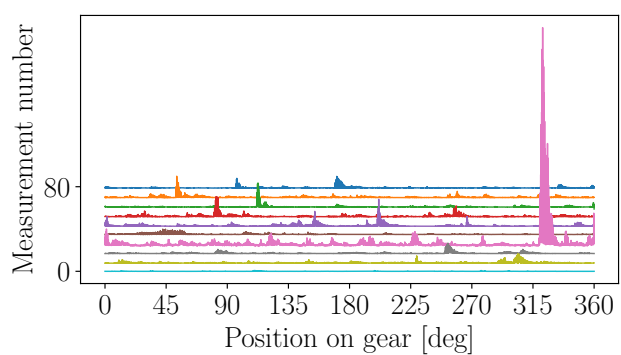

(b) SES: LGD

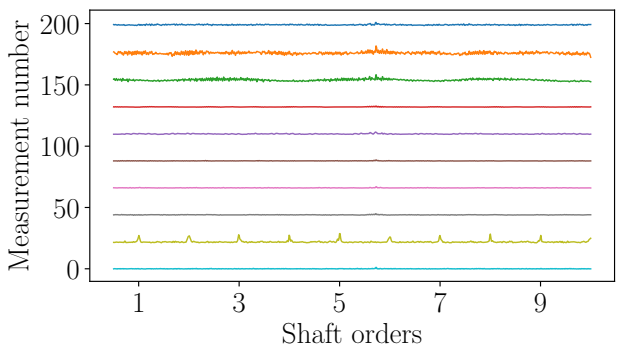

(d) SES: DGD

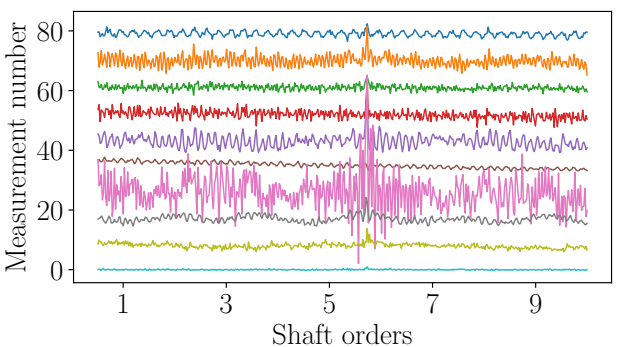

Figure 20: The Synchronous Average of the Squared Envelope (SASE) and the Squared Envelope Spectrum (SES), obtained by using the ICS2gram to design a bandpass filter, are presented for the Localised Gear Damage (LGD) and Distributed Gear Damage (DGD) investigations.

(a) Kurtogram

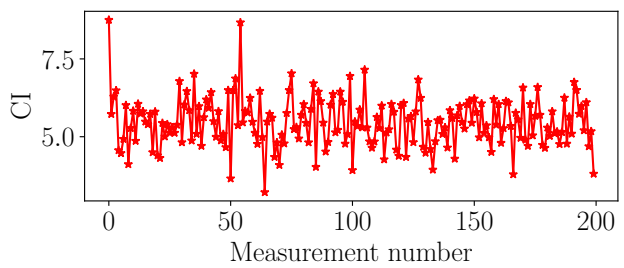

(b) ICS2gram

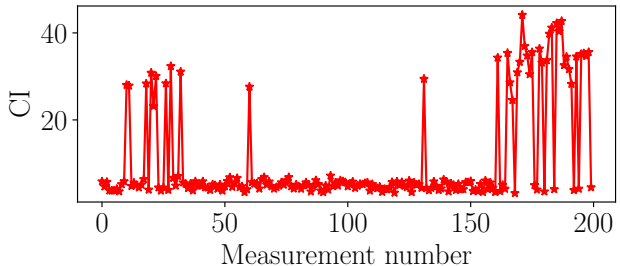

Figure 21: The Condition Indicators (CI), calculated with the bandlimited signals obtained with the Kurtogram and ICS2gram, are presented for the Localised Gear Damage (LGD) dataset.

method is on average between five and seven times slower than the conventional methods considered in this section, it performs much better on the signals considered.

\section{Conclusions}

In this work, the combination of an optimisation-based frequency band identification method and historical data from a healthy machine is proposed for novelty detection under time-varying operating conditions. The optimisation-based method ensures that the optimal frequency band is obtained for enhancing the fault information in the signal, whereafter the available historical data are utilised to calculate a threshold for automatic fault detection under time-varying operating conditions. The proposed 
method is compared against existing methods. The results indicate that the proposed method is capable of enhancing the damage in the signals; it makes it possible to determine the portion of the gear that is damaged and it allows for automatic fault detection under time-varying operating conditions.

\section{Acknowledgements}

The Eskom Power Plant Engineering Institute (EPPEI) is gratefully acknowledged for their support in the execution of this research.

\section{References}

[1] Y. Lin, L. Tu, H. Liu, W. Li, Fault analysis of wind turbines in China, Renewable and Sustainable Energy Reviews 55 (2016) 482-490.

[2] J. P. Salameh, S. Cauet, E. Etien, A. Sakout, L. Rambault, Gearbox condition monitoring in wind turbines: A review, Mechanical Systems and Signal Processing 111 (2018) 251-264.

[3] R. B. Randall, J. Antoni, Rolling element bearing diagnostics - A tutorial, Mechanical Systems and Signal Processing 25 (2) (2011) 485-520.

[4] A. Jablonski, Z. Dworakowski, K. Dziedziech, F. Chaari, Vibration-based diagnostics of epicyclic gearboxes-from classical to soft-computing methods, Measurement 147 (2019) 106811.

[5] S. Schmidt, P. S. Heyns, Normalisation of the amplitude modulation caused by time-varying operating conditions for condition monitoring, Measurement 149 (2020) 106964.

[6] S. Kass, A. Raad, J. Antoni, Self-running bearing diagnosis based on scalar indicator using fast order frequency spectral coherence, Measurement 138 (2019) 467-484.

[7] G. Yu, C. Li, J. Zhang, A new statistical modeling and detection method for rolling element bearing faults based on alpha-stable distribution, Mechanical Systems and Signal Processing 41 (1-2) (2013) 155-175.

[8] D. Abboud, J. Antoni, S. Sieg-Zieba, M. Eltabach, Envelope analysis of rotating machine vibrations in variable speed conditions: A comprehensive treatment, Mechanical Systems and Signal Processing 84 (2017) 200-226.

[9] S. Schmidt, P. S. Heyns, K. C. Gryllias, A pre-processing methodology to enhance novel information for rotating machine diagnostics, Mechanical Systems and Signal Processing 124 (2019) 541-561. 
[10] T. Barszcz, R. B. Randall, Application of spectral kurtosis for detection of a tooth crack in the planetary gear of a wind turbine, Mechanical Systems and Signal Processing 23 (4) (2009) 13521365.

[11] J. Antoni, R. B. Randall, The spectral kurtosis: Application to the vibratory surveillance and diagnostics of rotating machines, Mechanical Systems and Signal Processing 20 (2) (2006) 308-331.

[12] F. Combet, L. Gelman, Optimal filtering of gear signals for early damage detection based on the spectral kurtosis, Mechanical Systems and Signal Processing 23 (3) (2009) 652-668.

[13] J. Antoni, Fast computation of the kurtogram for the detection of transient faults, Mechanical Systems and Signal Processing 21 (1) (2007) 108-124.

[14] J. Antoni, The infogram: Entropic evidence of the signature of repetitive transients, Mechanical Systems and Signal Processing 74 (2016) 73-94.

[15] T. Barszcz, A. JabŁoński, A novel method for the optimal band selection for vibration signal demodulation and comparison with the kurtogram, Mechanical Systems and Signal Processing 25 (1) (2011) 431-451.

[16] Y. Lei, J. Lin, Z. He, Y. Zi, Application of an improved kurtogram method for fault diagnosis of rolling element bearings, Mechanical Systems and Signal Processing 25 (5) (2011) 1738-1749.

[17] D. Wang, P. W. Tse, K. L. Tsui, An enhanced Kurtogram method for fault diagnosis of rolling element bearings, Mechanical Systems and Signal Processing 35 (1-2) (2013) 176-199.

[18] W. A. Smith, Z. Fan, Z. Peng, H. Li, R. B. Randall, Optimised spectral kurtosis for bearing diagnostics under electromagnetic interference, Mechanical Systems and Signal Processing 75 (2016) 371-394.

[19] X. Xu, M. Zhao, J. Lin, Y. Lei, Envelope harmonic-to-noise ratio for periodic impulses detection and its application to bearing diagnosis, Measurement 91 (2016) 385-397.

[20] A. Moshrefzadeh, A. Fasana, The Autogram: An effective approach for selecting the optimal demodulation band in rolling element bearings diagnosis, Mechanical Systems and Signal Processing 105 (2018) 294-318.

[21] W. A. Smith, P. Borghesani, Q. Ni, K. Wang, Z. Peng, Optimal demodulation-band selection for envelope-based diagnostics: A comparative study of traditional and novel tools, Mechanical Systems and Signal Processing 134 (2019) 106303. 
[22] A. Mauricio, W. A. Smith, R. B. Randall, J. Antoni, K. Gryllias, Improved envelope spectrum via feature optimisation-gram (iesfogram): A novel tool for rolling element bearing diagnostics under non-stationary operating conditions, Mechanical Systems and Signal Processing 144 (2020) 106891.

[23] S. Schmidt, A. Mauricio, P. S. Heyns, K. C. Gryllias, A methodology for identifying information rich frequency bands for diagnostics of mechanical components-of-interest under time-varying operating conditions, Mechanical Systems and Signal Processing 142 (2020) 106739.

[24] J. Antoni, P. Borghesani, A statistical methodology for the design of condition indicators, Mechanical Systems and Signal Processing 114 (2019) 290-327.

[25] Q. Ni, K. Wang, J. Zheng, Rolling element bearings fault diagnosis based on a novel optimal frequency band selection scheme, IEEE Access 7 (2019) 80748-80766.

[26] J. Antoni, The spectral kurtosis: A useful tool for characterising non-stationary signals, Mechanical Systems and Signal Processing 20 (2) (2006) 282-307.

[27] Y. Zhang, R. B. Randall, Rolling element bearing fault diagnosis based on the combination of genetic algorithms and fast kurtogram, Mechanical Systems and Signal Processing 23 (5) (2009) 1509-1517.

[28] J. Wodecki, A. Michalak, R. Zimroz, Optimal filter design with progressive genetic algorithm for local damage detection in rolling bearings, Mechanical Systems and Signal Processing 102 (2018) 102-116.

[29] M. Buzzoni, J. Antoni, G. d'Elia, Blind deconvolution based on cyclostationarity maximization and its application to fault identification, Journal of Sound and Vibration 432 (2018) 569-601.

[30] J. S. Arora, Introduction to optimum design, Elsevier/Academic Press, 2004.

[31] The MathWorks, Inc., Optimization Toolbox, Natick, Massachusetts, United State (2020). URL https://www . mathworks.com/help/optim/

[32] P. Virtanen, R. Gommers, T. E. Oliphant, M. Haberland, T. Reddy, D. Cournapeau, E. Burovski, P. Peterson, W. Weckesser, J. Bright, S. J. van der Walt, M. Brett, J. Wilson, K. Jarrod Millman, N. Mayorov, A. R. J. Nelson, E. Jones, R. Kern, E. Larson, C. Carey, İ. Polat, Y. Feng, E. W. Moore, J. Vand erPlas, D. Laxalde, J. Perktold, R. Cimrman, I. Henriksen, E. A. Quintero, C. R. Harris, A. M. Archibald, A. H. Ribeiro, F. Pedregosa, P. van Mulbregt, S. . Contributors, SciPy 1.0: Fundamental Algorithms for Scientific Computing in Python, Nature Methods 17 (2020) 261-272. doi:https://doi.org/10.1038/s41592-019-0686-2. 
[33] Q. Leclère, H. André, J. Antoni, A multi-order probabilistic approach for Instantaneous Angular Speed tracking debrie fi ng of the CMMNO'14 diagnosis contest, Mechanical Systems and Signal Processing 81 (2016) 375-386.

[34] D. Abboud, S. Baudin, J. Antoni, D. Rémond, M. Eltabach, O. Sauvage, The spectral analysis of cyclo-non-stationary signals, Mechanical Systems and Signal Processing 75 (2016) 280-300.

[35] N. Pham, A. Malinowski, T. Bartczak, Comparative study of derivative free optimization algorithms, IEEE Transactions on Industrial Informatics 7 (4) (2011) 592-600.

[36] A. P. Engelbrecht, Computational Intelligence: An Introduction, 2nd Edition, Wiley Publishing, 2007.

[37] F. Gao, L. Han, Implementing the nelder-mead simplex algorithm with adaptive parameters, Computational Optimization and Applications 51 (1) (2012) 259-277.

[38] W. Wang, Early detection of gear tooth cracking using the resonance demodulation technique, Mechanical Systems and Signal Processing 15 (5) (2001) 887-903.

[39] P. McFadden, M. Toozhy, Application of Synchronous Averaging To Vibration Monitoring of Rolling Element Bearings, Mechanical Systems and Signal Processing 14 (6) (2000) 891-906.

[40] A. Raad, J. Antoni, M. Sidahmed, Indicators of cyclostationarity: Theory and application to gear fault monitoring, Mechanical Systems and Signal Processing 22 (3) (2008) 574-587.

[41] K. Gryllias, S. Moschini, J. Antoni, Application of cyclo-nonstationary indicators for bearing monitoring under varying operating conditions, Journal of Engineering for Gas Turbines and Power 140 (1). 